\title{
Thermodynamics of ferrofluids in applied magnetic fields
}

\author{
Ekaterina A. Elfimova and Alexey O. Ivanov \\ Institute of Mathematics and Computer Sciences, Ural Federal University, 51 Lenin Avenue, Ekaterinburg 620000, Russia
}

Philip J. Camp*

School of Chemistry, University of Edinburgh, West Mains Road, Edinburgh EH9 3JJ, Scotland

(Received 12 August 2013; published 23 October 2013)

\begin{abstract}
The thermodynamic properties of ferrofluids in applied magnetic fields are examined using theory and computer simulation. The dipolar hard sphere model is used. The second and third virial coefficients $\left(B_{2}\right.$ and $\left.B_{3}\right)$ are evaluated as functions of the dipolar coupling constant $\lambda$, and the Langevin parameter $\alpha$. The formula for $B_{3}$ for a system in an applied field is different from that in the zero-field case, and a derivation is presented. The formulas are compared to results from Mayer-sampling calculations, and the trends with increasing $\lambda$ and $\alpha$ are examined. Very good agreement between theory and computation is demonstrated for the realistic values $\lambda \leqslant 2$. The analytical formulas for the virial coefficients are incorporated in to various forms of virial expansion, designed to minimize the effects of truncation. The theoretical results for the equation of state are compared against results from Monte Carlo simulations. In all cases, the so-called logarithmic free energy theory is seen to be superior. In this theory, the virial expansion of the Helmholtz free energy is re-summed in to a logarithmic function. Its success is due to the approximate representation of high-order terms in the virial expansion, while retaining the exact low-concentration behavior. The theory also yields the magnetization, and a comparison with simulation results and a competing modified mean-field theory shows excellent agreement. Finally, the putative field-dependent critical parameters for the condensation transition are obtained and compared against existing simulation results for the Stockmayer fluid. Dipolar hard spheres do not undergo the transition, but the presence of isotropic attractions, as in the Stockmayer fluid, gives rise to condensation even in zero field. A comparison of the relative changes in critical parameters with increasing field strength shows excellent agreement between theory and simulation, showing that the theoretical treatment of the dipolar interactions is robust.
\end{abstract}

DOI: 10.1103/PhysRevE.88.042310 PACS number(s): 75.50.Mm, 05.20.Jj, 05.70.Ce, 64.10.+h

\section{INTRODUCTION}

Ferrofluids are dispersions of ferromagnetic grains suspended in a carrier liquid. The grain size is roughly $10 \mathrm{~nm}$ to sustain ferromagnetic order, and to avoid the formation of magnetic domains. The carrier liquid may be nonaqueous (in which case, the grains must be sterically stabilized) or aqueous (requiring charge stabilization). Typically, the concentration (or packing fraction) of magnetic grains is of order $0.01-0.1$ by volume. Much of the functionality of ferrofluids arises from the responses of the structure and dynamics to applied magnetic fields. For instance, the rheological properties (such as viscosity) and optical properties (such as birefringence) become anisotropic in the presence of a uniform magnetic field [1]. This arises due to the field-induced alignment of the particles and the enhancement of chain-like correlations in the field direction. This effect has been studied in detail in recent work [2]. Ferrofluids may be used in a variety of applications, such as in devices (sealants, heat-conduction media, media for hydraulic suspensions) and in materials processing (separation media, gas-fluidized beds). Such materials can also be adapted for biomedical uses such as targeted drug delivery, diagnosis, and localized treatment by hyperthermia.

Ferrofluids also provide a realization of a model polar liquid. If the magnetic grains are spherical and homogeneously magnetized, then the magnetic interaction between any two

*philip.camp@ed.ac.uk grains $i$ and $j$ is given by the pure dipole-dipole interaction

$$
u_{i j}^{\mathrm{d}}=\frac{\mu_{0}}{4 \pi}\left[\frac{\left(\boldsymbol{\mu}_{i} \cdot \boldsymbol{\mu}_{j}\right)}{r_{i j}^{3}}-\frac{3\left(\boldsymbol{\mu}_{i} \cdot \boldsymbol{r}_{i j}\right)\left(\boldsymbol{\mu}_{j} \cdot \boldsymbol{r}_{i j}\right)}{r_{i j}^{5}}\right],
$$

where $\boldsymbol{r}_{i j}$ is the center-center separation vector, $r_{i j}=\left|\boldsymbol{r}_{i j}\right|$, $\boldsymbol{\mu}_{i}$ is the dipole moment on particle $i$, and $\mu_{0}$ is the magnetic permeability of the background medium. In addition, there will be a short-range interaction arising from steric and/or charge stabilization. In many cases, this can be approximated by a hard-sphere potential, given by

$$
u_{i j}^{\mathrm{s}}=\left\{\begin{array}{ll}
\infty & r_{i j}<\sigma \\
0 & r_{i j} \geqslant \sigma
\end{array},\right.
$$

where $\sigma$ is the particle diameter. Detailed numerical comparisons between the magnetic properties of real ferrofluids and those of the (polydisperse) dipolar hard sphere (DHS) model show that, for all practical purposes, these interactions are sufficient [3,4].

Such simple models can be studied in detail using theory and computer simulations. The DHS model, in particular, has been studied intensively for almost five decades, but it still yields surprises. One of the most long-standing problems is the phase diagram in the absence of a magnetic field, and specifically, whether there is a colloidal vapor-liquid phase transition. The debate is not yet fully resolved. The Boltzmannweighted angle average of the interaction potential is, to leading order, attractive and short range $\left(\sim-r^{-6}\right)$, and hence a conventional condensation transition is to be anticipated [5]. Computer simulations show that a transition is precluded, or 
at least interrupted, by extensive association of the particles to form chains, rings, and branched structures [6,7]. Despite a heavy investment of computer time, the condensation transition in DHS fluids has not been observed directly in simulations of systems with sufficient size, and with suitable treatments of the long-range dipolar interactions [6-10]. Many attempts have been made to "sneak up" on a phase transition by simulating models that correspond to pure DHSs in some limit, but the transition always disappears before the limit is reached [11-17]. It has been shown theoretically, and with several different approaches, that chain formation may preclude fluid-fluid phase separation [18-21]. The question of a phase transition is somewhat academic, however, because if there is such a transition, it occurs only for very strongly interacting particles. The interaction strength is measured with the dipolar coupling constant, $\lambda=\mu_{0} \mu^{2} / 4 \pi \sigma^{3} k_{\mathrm{B}} T$, where $\mu=|\boldsymbol{\mu}|, k_{\mathrm{B}}$ is Boltzmann's constant, and $T$ is the temperature. Extrapolations of simulation data to the DHS limit indicate that the critical value is $\lambda \simeq 6$; for typical ferrofluids, the dipolar coupling constants are normally in the region of $\lambda=1$ or 2 . The particle concentration is given by $\rho=N / V$, where $N$ is the number of particles and $V$ is the system volume, or alternatively the volume fraction $\varphi=\rho v_{0}$ where $v_{0}=\pi \sigma^{3} / 6$ is the particle volume. The critical concentration appears to be in the region of $\varphi \simeq 0.05$ (or $\rho \sigma^{3} \simeq 0.1$ ).

To understand and predict theoretically the properties of real ferrofluids, such as diffusion and sedimentation, it is necessary to be able to calculate thermodynamic properties such as osmotic pressure and free energy as functions of the volume fraction $\varphi$ and the dipolar coupling constant $\lambda$. There are many theories of dipolar liquids, but among the most successful (for the thermodynamic properties of DHSs) is the thermodynamic perturbation theory (TPT) of Stell and co-workers [22,23]. In a recent study, the present authors constructed a new theory based on the virial expansion, which outperforms the TPT, but requires only the first few virial coefficients as input [24]. The theory is based on a logarithmic representation of the Helmholtz free energy, and hence it will be referred to as the logarithmic free energy (LFE) theory; it will be summarized briefly in Sec. II C. A critical comparison of these theories with the equations of state from simulations of DHSs and experimental sedimentation profiles of real ferrofluids $[25,26]$ shows that the LFE theory is superior over all realistic ranges of volume fraction and dipolar coupling constant. To understand processes such as magnetophoresis in an applied magnetic field, however, requires knowledge of how the virial coefficients vary with field strength. In a uniform magnetic field $\boldsymbol{H}$, the total interaction energy of the fluid is

$$
U=\frac{1}{2} \sum_{i=1}^{N} \sum_{i \neq j}^{N}\left(u_{i j}^{\mathrm{s}}+u_{i j}^{\mathrm{d}}\right)-\mu_{0} \sum_{i=1}^{N} \boldsymbol{\mu}_{i} \cdot \boldsymbol{H}
$$

The Langevin parameter $\alpha=\mu_{0} \mu H / k_{\mathrm{B}} T$ measures the importance of the Zeeman interaction as compared to the thermal energy. There have been many theoretical studies focused on the structure and possible phases of dipolar particles in applied fields, using integral equations [27-29], classical density functional theory (DFT) [28,30], and virial expansions [2,31]. As far as the authors are aware, though, there has not been a systematic study of the thermodynamic properties, such as the equation of state, of DHSs in an applied field.

In this work, the dependences of the second and third virial coefficients, $B_{2}$ and $B_{3}$, on $\lambda$ and $\alpha$ are determined analytically and numerically. The correct expressions for these coefficients are derived quite generally for a one-component system in an applied field: the formula for $B_{2}$ is the same as in the zero-field case; the formula for $B_{3}$, however, is different in an applied field. This is a surprising result which is not emphasized in standard texts, but which has been noticed before in the virial expansion for flexible molecules [32,33]. The analytical evaluation of the virial coefficients is based on an expansion in $\lambda$ and a representation in terms of appropriate functions of $\alpha$. Numerical results are obtained using the Mayer-sampling method introduced by Singh and Kofke [34]. Next, the analytical results are incorporated in to various virial-type expressions for the thermodynamic functions, including the so-called perturbed virial expansion (PVE) developed by Nezbeda and co-workers [35-37], and the present authors' LFE theory [24]. The theoretical results for the equation of state are compared critically against Monte Carlo (MC) simulation results, and it is demonstrated that the LFE theory is superior for DHSs in an applied magnetic field. Knowing the Helmholtz free energy as a function of the field strength, it is possible to calculate the magnetization, and theoretical predictions for this quantity will be compared against simulation results. Currently the best theory for the magnetization curve of dense ferrofluids is the second-order modified mean-field theory (MMF2) of Ivanov and Kuznetsova [38], which has been tested thoroughly against results from experiments and simulations $[3,4]$. The new LFE theory compares favorably with the simulation results and the MMF2 theory.

Almost all approaches based on virial expansions, perturbation theory, DFT, or integral equations predict the existence of a critical point, even for DHSs. Even though simulations in an applied field suggest that there is no transition in DHSs [39], the dependences of putative DHS critical parameters on $\alpha$ can be explored theoretically. This is worthwhile because other models of polar fluids undergo condensation even in zero field. For example, in the Stockmayer fluid, the particles interact via Lennard-Jones (LJ) and dipole-dipole potentials, and this model's vapor-liquid critical point has been determined precisely for a broad range of interaction parameters using computer simulations [40-52]. From the theoretical point of view, the presence of the soft core in the interaction complicates the picture considerably, but it can be treated by using a suitable reference hard-sphere fluid with a particle diameter that depends on the interaction parameters and the temperature [53]. The relative variations of the critical parameters with $\alpha$ from simulation [44-46] can easily be compared with the predictions for the DHS system. The phase diagram of the Stockmayer fluid in a field has been analyzed theoretically using classical DFT by Groh and Dietrich [30]. Although the theory does not predict the critical parameters accurately as compared to simulation, the relative variation with field strength is described very well. There are, however, some unusual features in the theoretical phase diagram which have not been observed in simulations or experiments, namely, the existence of a critical point associated with a transition between isotropic and ferromagnetic liquid phases. In this 
work, a comparison of relative critical parameters will be carried out using the LFE theory, and remarkably good agreement between simulation and theory will be demonstrated. A specific thermodynamic theory for Stockmayer fluids will be detailed in a future publication. Note that in the related dipolar soft sphere (DSS) fluid, the $r^{-6}$ attraction in the LJ potential is omitted: just as in the DHS fluid, a zero-field transition is thought not to exist [54-57].

This article is arranged as follows. In Sec. II, analytical results for the virial coefficients are derived, the LFE theory is outlined, and the Mayer-sampling and MC calculations are described. The results are presented in Sec. III, organized in terms of the virial coefficients, the equation of state, the magnetization, and the phase behavior. Section IV concludes the article.

\section{THEORY AND SIMULATION}

\section{A. Virial coefficients for a system in an applied field}

The derivation of the virial coefficients is summarized in this section, with the aim of confirming the correct formulas for $B_{2}$ and $B_{3}$ in the case of a one-component system subjected to an applied field. The formula for $B_{3}$ differs from that in the textbook case of zero field; the case of a nonzero field does not appear to have been covered in any standard reference. It turns out that the correct expression for $B_{3}$ contains an extra term which has also been found in the third virial coefficient for flexible molecules: the so-called "uniquely flexible" contribution [32,33,58]. The common feature appears to be the presence of intramolecular degrees of freedom contributing to the potential energy, which in the present case corresponds to the dipolar orientation coupling to the applied field. For completeness, a full derivation is given here, the structure of which follows closely Secs. 23 and 25 of Ref. [59]. Consider a system comprising a volume $V$ at fixed temperature $T$ coupled to a particle reservoir with chemical potential $\mu$. Define the $N$-particle canonical partition function as

$$
\begin{aligned}
Q_{N}= & \frac{1}{N ! \mathcal{V}^{N}} \int d \boldsymbol{r}_{1} d \boldsymbol{\Omega}_{1} \cdots \int d \boldsymbol{r}_{N} d \boldsymbol{\Omega}_{N} \\
& \times \exp \left(-\beta \sum_{i<j}^{N} u_{i j}-\beta \sum_{i=1}^{N} \psi_{i}\right),
\end{aligned}
$$

where $\mathcal{V}$ is the temperature-dependent de Broglie thermal volume resulting from integrating the Boltzmann factor for the kinetic energy over linear and angular momenta, $\beta=1 / k_{\mathrm{B}} T$, $\boldsymbol{r}$ is the particle position, $\boldsymbol{\Omega}$ is the particle orientation, and $u_{i j}$ is the interaction potential. $\psi$ is a single-particle potential energy arising from an external field which is assumed only to act on the orientation; if the field acted on the particle position, then the system would be inhomogeneous and a different approach to the virial expansion would be required (such as DFT). The grand-canonical partition function is

$$
\Xi=\sum_{N=0}^{\infty} Q_{N} \exp (\beta \mu N)
$$

By writing $Q_{N}$ in the form

$$
Q_{N}=\left(\frac{\Psi}{\mathcal{V}}\right)^{N} \frac{Z_{N}}{N !}
$$

with

$$
\Psi=\int d \boldsymbol{\Omega} e^{-\beta \psi}
$$

the activity may be defined as $z=(\Psi / \mathcal{V}) \exp (\beta \mu)$, and hence the grand-canonical partition function becomes

$$
\Xi=\sum_{N=0}^{\infty} \frac{z^{N} Z_{N}}{N !}
$$

$Z_{N}$ is the configurational integral and is defined by Eq. (6). The pressure is related to $\Xi$ by $\beta P V=\ln \Xi$. An activity expansion for $\beta P V$ may be written

$$
\beta P V=\ln \Xi=\sum_{j \geqslant 1}^{\infty} V b_{j} z^{j} .
$$

Expanding $\Xi=\exp \left(\sum_{j \geqslant 1}^{\infty} V b_{j} z^{j}\right)$ in a Maclaurin series and matching terms of equal order in $z$ give the following results for $b_{j}$ :

$$
\begin{gathered}
b_{1}=\frac{Z_{1}}{V}=1, \\
b_{2}=\frac{1}{2 V}\left(Z_{2}-Z_{1}^{2}\right), \\
b_{3}=\frac{1}{6 V}\left(Z_{3}-3 Z_{2} Z_{1}+2 Z_{1}^{3}\right) .
\end{gathered}
$$

The average concentration is obtained from

$$
\rho=\frac{\langle N\rangle}{V}=\frac{z}{V} \frac{\partial \ln \Xi}{\partial z}=\sum_{j \geqslant 1}^{\infty} j b_{j} z^{j} .
$$

To get $P$ as a function of $\rho$, insert an expansion $z=a_{1} \rho+$ $a_{2} \rho^{2}+a_{3} \rho^{3}+\cdots$ into Eq. (13) to obtain

$$
\begin{aligned}
\rho= & b_{1} a_{1} \rho+\left(b_{1} a_{2}+2 b_{2} a_{1}^{2}\right) \rho^{2} \\
& +\left(b_{1} a_{3}+4 b_{2} a_{1} a_{2}+3 b_{3} a_{1}^{3}\right) \rho^{3}+\cdots,
\end{aligned}
$$

which implies that $a_{1}=1, a_{2}=-2 b_{2}$, and $a_{3}=-3 b_{3}+$ $8 b_{2}^{2}$. The expansion $z=\rho-2 b_{2} \rho^{2}+\left(8 b_{2}^{2}-3 b_{3}\right) \rho^{3}$ is then inserted in Eq. (9) to yield

$$
\begin{aligned}
\beta P & =\rho-b_{2} \rho^{2}+\left(4 b_{2}^{2}-2 b_{3}\right) \rho^{3}+\cdots \\
& =\rho\left(1+\sum_{n=1}^{\infty} B_{n+1} \rho^{n}\right) .
\end{aligned}
$$

Now the first two virial coefficients can be identified as follows:

$$
\begin{gathered}
B_{2}=-b_{2}, \\
B_{3}=4 b_{2}^{2}-2 b_{3} .
\end{gathered}
$$

To complete the calculations, $Z_{1}, Z_{2}$, and $Z_{3}$ are expressed in terms of the Mayer $f$ functions, $f_{i j}=\exp \left(-\beta u_{i j}\right)-1$. Translational invariance is assumed throughout, so that the position of any particle can be chosen as the origin of the coordinate system, and integrations can be performed over 
$\boldsymbol{r}_{i j}=\boldsymbol{r}_{j}-\boldsymbol{r}_{i}:$

$$
\begin{gathered}
Z_{1}=\frac{1}{\Psi} \int d 1 e^{-\beta \psi_{1}}=V, \\
Z_{2}=\frac{1}{\Psi^{2}} \int d 1 \int d 2\left(f_{12}+1\right) e^{-\beta\left(\psi_{1}+\psi_{2}\right)} \\
=V \int d \boldsymbol{r}_{12}\left\langle f_{12}\right\rangle+V^{2}, \\
Z_{3}=\frac{1}{\Psi^{3}} \int d 1 \int d 2 \int d 3\left(f_{12}+1\right)\left(f_{13}+1\right) \\
\times\left(f_{23}+1\right) e^{-\beta\left(\psi_{1}+\psi_{2}+\psi_{3}\right)} \\
=V \int d \boldsymbol{r}_{12} \int d \boldsymbol{r}_{13}\left\langle f_{12} f_{13} f_{23}\right\rangle+3 V \int d \boldsymbol{r}_{12} \\
\times \int d \boldsymbol{r}_{13}\left\langle f_{12} f_{13}\right\rangle+3 V^{2} \int d \boldsymbol{r}_{12}\left\langle f_{12}\right\rangle+V^{3} .
\end{gathered}
$$

In these expressions, $\int d i=\int d \boldsymbol{r}_{i} \int \mathrm{d} \boldsymbol{\Omega}_{i}$, and $\langle\cdots\rangle$ means a Boltzmann-weighted average over the orientation of each of the particles involved, e.g.,

$$
\left\langle f_{12}\right\rangle=\frac{1}{\Psi^{2}} \int d \boldsymbol{\Omega}_{1} \int d \boldsymbol{\Omega}_{2} f_{12} e^{-\beta\left(\psi_{1}+\psi_{2}\right)} .
$$

Equations (18)-(20) can be inserted into Eqs. (11) and (12) to give the following explicit expressions for $b_{2}$ and $b_{3}$ :

$$
\begin{gathered}
b_{2}=\frac{1}{2} \int d \boldsymbol{r}_{12}\left\langle f_{12}\right\rangle, \\
b_{3}=b_{3 a}+b_{3 b}, \\
b_{3 a}=\frac{1}{6} \int d \boldsymbol{r}_{12} \int d \boldsymbol{r}_{13}\left\langle f_{12} f_{13} f_{23}\right\rangle, \\
b_{3 b}=\frac{1}{2} \int d \boldsymbol{r}_{12} \int d \boldsymbol{r}_{13}\left\langle f_{12} f_{13}\right\rangle .
\end{gathered}
$$

The formula for $B_{2}=-b_{2}$ is the same as in the zero-field case. The complete formula for $B_{3}$ may be written in the transparent form

$$
\begin{gathered}
B_{3}=B_{3 a}+B_{3 b}, \\
B_{3 a}=-2 b_{3 a}=-\frac{1}{3} \int d \boldsymbol{r}_{12} \int d \boldsymbol{r}_{13}\left\langle f_{12} f_{13} f_{23}\right\rangle, \\
B_{3 b}=4 b_{2}^{2}-2 b_{3 b}=\int d \boldsymbol{r}_{12} \int d \boldsymbol{r}_{13}\left[\left\langle f_{12}\right\rangle\left\langle f_{13}\right\rangle-\left\langle f_{12} f_{13}\right\rangle\right] .
\end{gathered}
$$

In zero field $(\alpha=0)$ or in an infinite field $(\alpha=\infty), B_{3 b}=0$ and hence $B_{3}=B_{3 a}$, the normally quoted result. In a finite applied field, $B_{3 b} \neq 0$ because the orientational averaging over $\boldsymbol{\Omega}_{2}$ couples $f_{12}$ and $f_{13}$, and hence $\left\langle f_{12}\right\rangle\left\langle f_{13}\right\rangle \neq\left\langle f_{12} f_{13}\right\rangle$.

\section{B. Virial coefficients for DHSs in an applied field}

Consider $N$ DHSs confined to a volume $V$ and temperature $T$. For particle $i$, the particle position is given by $\boldsymbol{r}_{i}$, and the orientation of the dipole $\boldsymbol{\mu}_{i}=\mu \boldsymbol{\Omega}_{i}$ is described by the unit vector $\boldsymbol{\Omega}_{i}=\left(\sin \omega_{i} \cos \zeta_{i}, \sin \omega_{i} \sin \zeta_{i}, \cos \omega_{i}\right)$. The system is exposed to an external magnetic field denoted by $\boldsymbol{H}$, which will be parallel to the laboratory $z$ direction, so that $\beta \psi_{i}=$ $-\beta \mu_{0} \mu_{i} \cdot \boldsymbol{H}=-\alpha \cos \omega_{i}$. In this case, $\Psi=4 \pi \sinh (\alpha) / \alpha$, and averages such as Eq. (21) can be written

$$
\left\langle f_{12}\right\rangle=\left(\frac{\alpha}{4 \pi \sinh \alpha}\right)^{2} \int d \boldsymbol{\Omega}_{1} \int d \boldsymbol{\Omega}_{2} f_{12} e^{\alpha \cos \omega_{1}+\alpha \cos \omega_{2}}
$$

The virial coefficients are evaluated as expansions in the dipolar coupling constant $\lambda$ up to third order. This is achieved by writing the Mayer $f$ function in the form

$$
f_{i j}=f_{i j}^{\mathrm{s}}+\left(f_{i j}^{\mathrm{s}}+1\right) f_{i j}^{\mathrm{d}}=f_{i j}^{\mathrm{s}}+\left(f_{i j}^{\mathrm{s}}+1\right) \sum_{k=1}^{\infty} \frac{\left(-\beta u_{i j}^{\mathrm{d}}\right)^{k}}{k !},
$$

where $f_{i j}^{\mathrm{s}}$ and $f_{i j}^{\mathrm{d}}$ are the Mayer $f$ functions for the short-range and dipolar interactions, respectively, and each factor of $\beta u_{i j}^{\mathrm{d}}$ yields an additional power of $\lambda$. Some of the terms depend on sample geometry. Throughout this work, it is assumed that the sample is cylindrical and of infinite elongation, in which case the demagnetization fields vanish. These calculations are laborious, and the details are given in the Supplemental Material [60]. The second virial coefficient is given by

$$
\begin{aligned}
\frac{B_{2}}{B_{2}^{\mathrm{HS}}}= & 1-\lambda L^{2}(\alpha)-\frac{\lambda^{2}}{3}\left[1+\frac{L_{3}^{2}(\alpha)}{5}\right] \\
& -\frac{\lambda^{3}}{105}\left[\frac{2 L(\alpha) L_{3}(\alpha)}{\alpha}-\frac{5 L_{3}^{2}(\alpha)}{\alpha^{2}}+4 L^{2}(\alpha)\right],
\end{aligned}
$$

where $B_{2}^{\mathrm{HS}}=4 v_{0}$ is the result for hard spheres, $L(\alpha)=$ $\operatorname{coth}(\alpha)-\alpha^{-1}$ is the Langevin function, and $L_{n}(\alpha)=1-$ $n L(\alpha) / \alpha$ with limits $L_{n}(0)=1-n / 3$ and $L_{n}(\infty)=1$. In zero field, $B_{2}$ depends only on even powers of $\lambda$ [24]: at the current level of approximation, $B_{2}(\alpha=0)=B_{2}^{\mathrm{HS}}\left(1-\lambda^{2} / 3\right)$. In an applied field, odd powers of $\lambda$ appear; in an infinitely strong field, $B_{2}(\alpha=\infty)=B_{2}^{\mathrm{HS}}\left(1-\lambda-2 \lambda^{2} / 5-4 \lambda^{3} / 105\right)$. The third virial coefficient is considerably more complex. Separate expressions for $B_{3 a}$ and $B_{3 b}$ are given in the Supplemental Material [60], and the complete expression for $B_{3}$ is

$$
\begin{aligned}
\frac{B_{3}}{B_{3}^{\mathrm{HS}}}= & 1-\frac{\lambda^{2}}{5}\left\{\left(2 \ln 2+\frac{1}{3}\right)\left[1+\frac{L_{3}^{2}(\alpha)}{5}\right]+2 L^{2}(\alpha)\left[-16 L^{2}(\alpha)-\frac{29 L(\alpha)}{\alpha}+10\right]\right\}-\frac{\lambda^{3}}{5}\left[\left(\frac{292}{5}+\frac{12}{5} \ln 2\right) \frac{L^{3}(\alpha)}{\alpha}\right. \\
& +(288 \ln 2-174) \frac{L^{3}(\alpha)}{\alpha^{3}}-\frac{64}{15} L^{2}(\alpha) L_{3}^{2}(\alpha)+\left(\frac{112}{5}+\frac{48}{35} \ln 2\right) L^{2}(\alpha) L_{3}(\alpha)+(192 \ln 2-146) \frac{L^{2}(\alpha) L_{3}(\alpha)}{\alpha^{2}} \\
& +(48-96 \ln 2) \frac{L^{2}(\alpha)}{\alpha^{2}}-\frac{3686}{175} L^{2}(\alpha)+\frac{20 L(\alpha) L_{2}(\alpha) L_{3}(\alpha)}{\alpha}+\left(\frac{1884}{7} \ln 2-188\right) \frac{L(\alpha) L_{3}^{2}(\alpha)}{\alpha}+\frac{71}{525} \frac{L(\alpha) L_{3}(\alpha)}{\alpha} \\
& \left.+(48-96 \ln 2) L_{2}^{2}(\alpha) L_{3}(\alpha)+\left(\frac{232}{5}-\frac{384}{5} \ln 2\right) L_{2}(\alpha) L_{3}^{2}(\alpha)+\left(\frac{864}{5} \ln 2-\frac{3384}{35}\right) L_{3}^{3}(\alpha)-\frac{71}{210} \frac{L_{3}^{2}(\alpha)}{\alpha^{2}}\right] .
\end{aligned}
$$


The hard-sphere value is $B_{3}^{\mathrm{HS}}=10 v_{0}^{2}$. Note that there is no term linear in $\lambda$, irrespective of the field strength (see the Supplemental Material for an explanation) [60]. The zero-field and infinite-field limits are

$$
\begin{aligned}
B_{3}(\alpha=0)=B_{3}^{\mathrm{HS}} & {\left[1-\frac{\lambda^{2}}{5}\left(2 \ln 2+\frac{1}{3}\right)+\frac{2 \lambda^{3}}{9}\right], } \\
B_{3}(\alpha=\infty)= & B_{3}^{\mathrm{HS}}\left[1-\frac{2 \lambda^{2}}{25}(6 \ln 2-29)\right. \\
& \left.-\frac{2 \lambda^{3}}{175}\left(24 \ln 2-\frac{1369}{15}\right)\right] .
\end{aligned}
$$

\section{Thermodynamics}

The virial expansion of the compressibility factor $Z$ is

$$
Z=\frac{P V}{N k_{\mathrm{B}} T}=1+\sum_{n=1}^{\infty} B_{n+1} \rho^{n},
$$

where $P$ is the (osmotic) pressure. This expansion in $\rho$ is only slowly convergent, if at all [53]. Several different strategies have been proposed to avoid this problem. Nezbeda and co-workers proposed a so-called perturbed virial expansion (PVE) in those cases where the equation of state of a reference system is known accurately [35]. In the present context, the reference system is the hard-sphere (HS) fluid, and the system of interest is the DHS fluid. In this case, the PVE is the formally exact relation

$$
Z=Z^{\mathrm{HS}}+\sum_{n=1}^{\infty} \Delta B_{n+1} \rho^{n},
$$

where $Z^{\mathrm{HS}}$ is the compressibility factor of the reference HS fluid, and $\Delta B_{n}=B_{n}-B_{n}^{\mathrm{HS}}$. $Z^{\mathrm{HS}}$ is known in the accurate and convenient Carnahan-Starling form [61]:

$$
Z^{\mathrm{HS}}=\frac{1+\varphi+\varphi^{2}-\varphi^{3}}{(1-\varphi)^{3}} .
$$

An approximate PVE expression with terms up to $\Delta B_{3} \rho^{2}$ has been shown to give good results for the critical parameters of the square-well fluid [36]. In Ref. [24], a new theory was developed from a logarithmic representation of the Helmholtz free energy $F$. For the case of DHSs in an applied field, this theory is based on the relation

$$
\begin{aligned}
\frac{\beta F}{N}-\frac{\beta F^{\mathrm{HS}}}{N}+\ln \Psi & =\sum_{n=1}^{\infty} n^{-1} \Delta B_{n+1} \rho^{n} \\
& =-\ln \left(1+\sum_{n=1}^{\infty} n^{-1} I_{n+1} \rho^{n}\right),
\end{aligned}
$$

where $F^{\mathrm{HS}}$ is the Helmholtz free energy for hard spheres

$$
\frac{\beta F^{\mathrm{HS}}}{N}=\ln (\rho \mathcal{V})-1+\frac{\varphi(4-3 \varphi)}{(1-\varphi)^{2}},
$$

and the first two coefficients $I_{2}$ and $I_{3}$ in Eq. (38) are

$$
\begin{gathered}
I_{2}=-\Delta B_{2}, \\
I_{3}=-\Delta B_{3}+\Delta B_{2}^{2},
\end{gathered}
$$

which can be confirmed by a Maclaurin expansion. Including these terms, the equation of state is given by

$$
Z=\rho \frac{\partial}{\partial \rho}\left(\frac{\beta F}{N}\right)=Z^{\mathrm{HS}}-\frac{I_{2} \rho+I_{3} \rho^{2}}{1+I_{2} \rho+\frac{1}{2} I_{3} \rho^{2}} .
$$

The potential advantages of the LFE expressions for $F$ and $Z$ are that they should be less sensitive to truncation of the sums, they always retain the exact low-order virial expansion, and they generate higher-order terms in $\rho$. In Ref. [24] it was shown that the LFE expressions containing expansions of $B_{2}$, $B_{3}$, and $B_{4}$ in terms of $\lambda$ are superior to the traditional TPT results $[22,23]$ for DHS fluids with $\lambda \leqslant 4$ and $\rho \sigma^{3} \leqslant 0.95$.

\section{Numerical methods}

The second virial coefficient was calculated numerically using the Mayer-sampling technique devised by Singh and Kofke [34]. The idea behind Mayer sampling is to evaluate the ratio of the cluster integrals for the potential of interest and a reference potential (in this case, hard spheres). For example, the ratio of the second virial coefficients for the DHS and HS fluids is

$$
\frac{B_{2}}{B_{2}^{\mathrm{HS}}}=\frac{\left\langle f_{12} e^{\alpha\left(\cos \omega_{1}+\cos \omega_{2}\right)} / w\right\rangle_{w}}{\left\langle f_{12}^{\mathrm{s}} e^{\alpha\left(\cos \omega_{1}+\cos \omega_{2}\right)} / w\right\rangle_{w}},
$$

where $w$ is a weight with which particle configurations are selected, and $\langle\cdots\rangle_{w}$ denotes an average over this "biased" distribution of configurations. The efficiency of the method relies in importance sampling of those particle positions and orientations that make the largest contributions to the virial coefficient for the system of interest, and this is achieved using the standard Metropolis algorithm. The scheme detailed in Ref. [34] is followed here, e.g., for the calculation of $B_{2}$, $w=\left|f_{12} e^{\alpha\left(\cos \omega_{1}+\cos \omega_{2}\right)}\right|$.

$B_{2}$ in a field depends on sample geometry. Expanding the Mayer function [Eq. (30)] gives

$$
\begin{aligned}
f_{i j} & =f_{i j}^{\mathrm{s}}-\left(f_{i j}^{\mathrm{s}}+1\right) \beta u_{i j}^{\mathrm{d}}+\frac{1}{2}\left(f_{i j}^{\mathrm{s}}+1\right)\left(\beta u_{i j}^{\mathrm{d}}\right)^{2}+\cdots \\
& \equiv-\left(f_{i j}^{\mathrm{s}}+1\right) \beta u_{i j}^{\mathrm{d}}+\Delta f_{i j},
\end{aligned}
$$

which isolates one term of order $\beta u_{i j}^{\mathrm{d}} \sim r_{i j}^{-3}$ which depends on sample geometry; each of the terms in the remainder $\left(\Delta f_{i j}\right)$ is of shorter range and does not cause any problems. The long-range contribution to $B_{2}$ has already been evaluated as in the second term of Eq. (31):

$$
\frac{1}{2} \int\left\langle\beta u_{12}^{\mathrm{d}}\right\rangle\left(f_{12}^{\mathrm{s}}+1\right) d \boldsymbol{r}_{12}=-4 v_{0} \lambda L^{2}(\alpha) .
$$

Mayer sampling is used to evaluate all of the remaining contributions giving

$$
B_{2}=-\frac{1}{2} \int\left\langle\Delta f_{12}\right\rangle d \boldsymbol{r}_{12}-4 v_{0} \lambda L^{2}(\alpha) .
$$

Unfortunately, a straightforward application of this approach to $B_{3}$ is not possible. $B_{3 a}$ in Eq. (27) can be computed easily enough, as there is no term in a $\lambda$ expansion of $f_{12} f_{13} f_{23}$ that depends on sample geometry. $B_{3 b}$, on the other hand, causes problems because there are contributions at all orders in $\lambda$ that do depend on sample geometry. Inserting Eq. (44) into Eq. (28) 
and collecting equivalent terms gives

$$
\begin{aligned}
B_{3 b}= & \int d \boldsymbol{r}_{12} \int d \boldsymbol{r}_{13}\left\{( f _ { 1 2 } ^ { \mathrm { s } } + 1 ) ( f _ { 1 3 } ^ { \mathrm { s } } + 1 ) \left[\left\langle\beta u_{12}^{\mathrm{d}}\right\rangle\left\langle\beta u_{13}^{\mathrm{d}}\right\rangle\right.\right. \\
& \left.-\left\langle\left(\beta u_{12}^{\mathrm{d}}\right)\left(\beta u_{13}^{\mathrm{d}}\right)\right\rangle\right]+2\left(f_{12}^{\mathrm{s}}+1\right)\left[\left\langle\beta u_{12}^{\mathrm{d}} \Delta f_{13}\right\rangle\right. \\
& \left.\left.-\left\langle\beta u_{12}^{\mathrm{d}}\right\rangle\left\langle\Delta f_{13}\right\rangle\right]+\left[\left\langle\Delta f_{12}\right\rangle\left\langle\Delta f_{13}\right\rangle-\left\langle\Delta f_{12} \Delta f_{13}\right\rangle\right]\right\} .
\end{aligned}
$$

The terms $\left\langle\beta u_{12}^{\mathrm{d}}\right\rangle$ and $\left\langle\left(\beta u_{12}^{\mathrm{d}}\right)\left(\beta u_{13}^{\mathrm{d}}\right)\right\rangle$ can be evaluated and integrated exactly. (See the calculations of $A_{1}$ and $A_{4}$ in the Supplemental Material [60].) The terms involving $\left\langle\Delta f_{12}\right\rangle$ and $\left\langle\Delta f_{12} \Delta f_{13}\right\rangle$ can be determined by Mayer sampling. The term $\left\langle\beta u_{12}^{\mathrm{d}} \Delta f_{13}\right\rangle$ cannot be evaluated exactly: by further expansion of $\Delta f_{13}$ in terms of $\beta u_{13}^{\mathrm{d}}$, there are contributions like $\left\langle\left(\beta u_{12}^{\mathrm{d}}\right)\left(\beta u_{13}^{\mathrm{d}}\right)^{k}\right\rangle$ at all orders $k \geqslant 1$, and each one of those depends on sample geometry because $\beta u_{12}^{\mathrm{d}} \sim r_{12}^{-3}$. The complete sum of these terms cannot be integrated analytically or by Mayer sampling. Shaul et al. present an "alternate root" formulation of a term equivalent to $B_{3 b}$, in order to facilitate Mayer-sampling calculations for flexible molecules [58]. This involves including a fourth particle at the origin, at the same position as the original "root" particle. With dipolar interactions, this formulation suffers from the same problem as that above; essentially, it arises from integrating over the product of two Mayer $f$ functions. Since a simple correction as in Eq. (46) is not available, a comparison of the analytical and Mayer-sampling results for $B_{3 a}$ will be presented instead.

Finally, bulk equations of state were calculated using conventional isothermal-isobaric Monte Carlo (NPT-MC) simulations of $N=256$ particles in a cubic simulation cell with periodic boundary conditions applied [62]. The longrange dipolar interactions were evaluated using the Ewald summation with conducting boundary conditions, for which the demagnetization fields are zero, consistent with the analytical results reported in Sec. IIB and derived in the Supplemental Material [60]. Each MC sweep consisted of an attempted translation or rotation for each of $N$ randomly selected particles, and one attempted volume move. For each state point, the system was equilibrated for $1 \times 10^{5} \mathrm{MC}$ sweeps, and a production run consisted of $4 \times 10^{5} \mathrm{MC}$ sweeps. Maximum translation, rotation, and volume displacements were adjusted to give acceptance rates of $20 \%, 50 \%$, and $20 \%$, respectively.

\section{RESULTS}

In the following, special attention is focused on DHS fluids with dipolar coupling constants $\lambda=1$ and 2 . These values are actually quite high for typical ferrofluids, although recent experimental measurements of osmotic equations of state show that values of $\lambda \simeq 2$ are achievable $[25,26]$.

\section{A. Virial coefficients}

Figure 1(a) shows the second virial coefficients $B_{2}$ for DHS fluids with $\lambda=1$ and $\lambda=2$, as functions of the Langevin parameter $\alpha$. Results are shown from analytical theory [Eq. (31)] and Mayer sampling [Eq. (46)]. The stronger the attractive interactions between particles, the lower the virial coefficient. The results show that the introduction of an applied
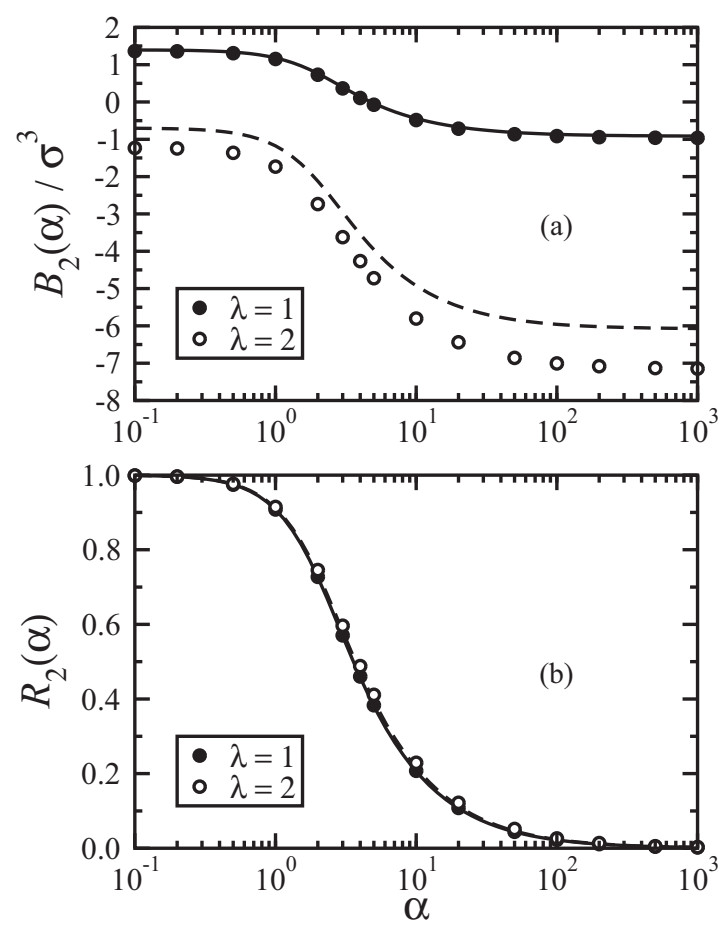

FIG. 1. The second virial coefficient $B_{2}(\alpha)$ as a function of the Langevin parameter $\alpha$ from theory (lines) and Mayer sampling (points): (a) absolute values; (b) relative values $R_{2}(\alpha)=\left[B_{2}(\alpha)-\right.$ $\left.B_{2}(\infty)\right] /\left[B_{2}(0)-B_{2}(\infty)\right]$. Data are shown for dipolar coupling constants $\lambda=1$ (solid line and filled points) and $\lambda=2$ (dashed line and open points).

field leads to an enhancement of attractive interactions. The field aligns the dipoles in parallel orientations, and the contributions to $f_{12}$ from the "nose-to-tail" parallel conformation are dominant, leading to a decrease in $B_{2}$. For $\lambda=1$, the agreement between theory and computation is excellent, with there being no discernible discrepancy. For $\lambda=2$ there is a deviation of roughly $1 \sigma^{3}$ at high values of $\alpha$, and smaller deviations at lower values of $\alpha$. Clearly this arises from the truncation of the expansion in $\lambda$ in Eq. (31). Higher order terms in this expansion are not easy to calculate, even in zero applied field [24]. Figure 1(b) shows that the relative variation with $\alpha$ is captured almost perfectly by theory; the plot of $R_{2}(\alpha)=\left[B_{2}(\alpha)-B_{2}(\infty)\right] /\left[B_{2}(0)-B_{2}(\infty)\right]$ shows almost perfect agreement between theory and computation.

Results for $B_{3 a}, B_{3 b}$, and $B_{3}$ for DHS fluids with $\lambda=1$ and $\lambda=2$ are shown in Fig. 2. Theory yields a complete expression for $B_{3}$, while Mayer sampling can only be used to evaluate $B_{3 a}$. First, theory shows that as the field is increased, $B_{3}$ increases and the total interaction between three particles become more repulsive. Although the field aligns the dipoles in parallel orientations, there are more "repulsive" threeparticle configurations than "attractive" ones. An attractive configuration might consist of three particles aligned with the field in a chain, but there are relatively few such arrangements. Second, $B_{3 b}=0$ for $\alpha=0$ and $\alpha=\infty$, as explained at the end of Sec. II A. At intermediate values of $\alpha$, the difference is still rather small, but it is maximal at $\alpha \simeq 2-3$. Finally, the agreement between $B_{3 a}$ from theory and computation is excellent for $\lambda=1$, and qualitative for $\lambda=2$. The $\alpha$ 

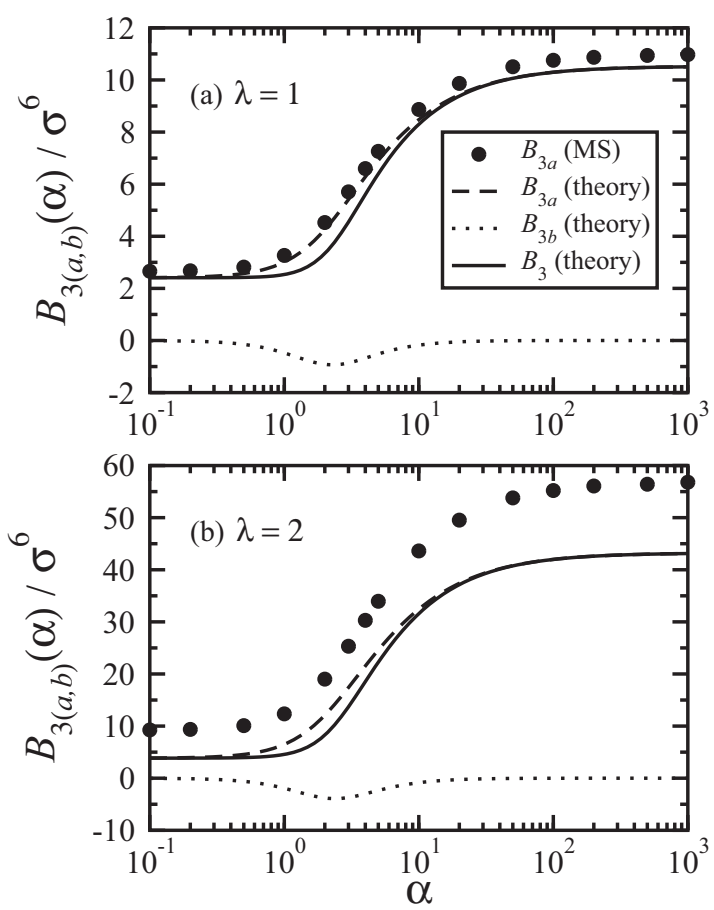

FIG. 2. Contributions to the third virial coefficient $B_{3}(\alpha)$ as functions of the Langevin parameter $\alpha$ from theory (lines) and Mayer sampling (MS) (points) at (a) $\lambda=1$ and (b) $\lambda=2$. The points are $B_{3 a}$ calculated by Mayer sampling, the dashed lines are $B_{3 a}$ calculated from theory, the dotted lines are $B_{3 b}$ calculated from theory, and the solid lines are $B_{3}$ calculated from theory.

dependence is captured extremely well by theory, even if the truncation of the expansion in $\lambda$ compromises the quantitative accuracy. A plot of the relative change in $B_{3 a}$, by analogy with Fig. 1(b), shows equally good agreement between theory and computation. (Data not shown for brevity.)

The dependences of $B_{2}$ and $B_{3}$ on $\lambda$ at fixed values of $\alpha$ in the range $0 \leqslant \alpha \leqslant \infty$ are shown in Figs. 3 and 4 , respectively. As already stated, the theoretical expression for $B_{2}$ does very well for $\lambda=1$ and at all values of $\alpha$, with almost

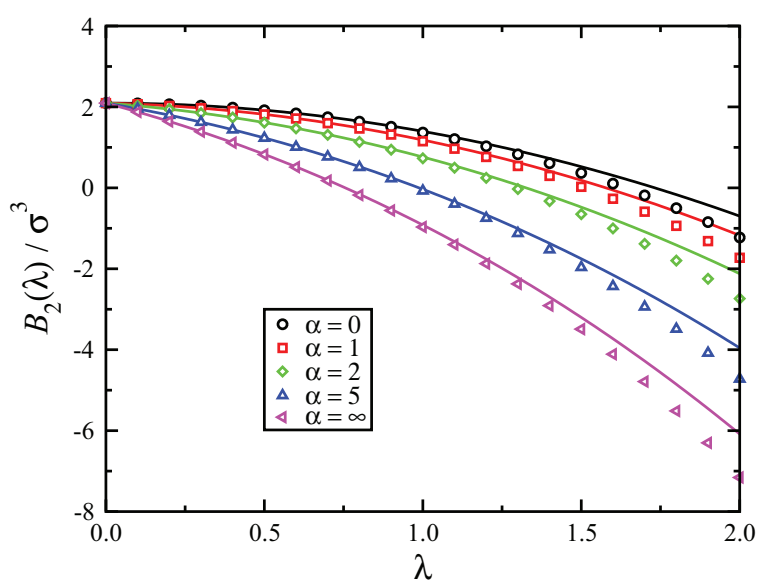

FIG. 3. (Color online) The second virial coefficient $B_{2}(\lambda)$ as a function of the dipolar coupling constant $\lambda$ from theory (lines) and Mayer sampling (points). Data are shown for Langevin parameters $\alpha=0,1,2,5$, and $\infty$.

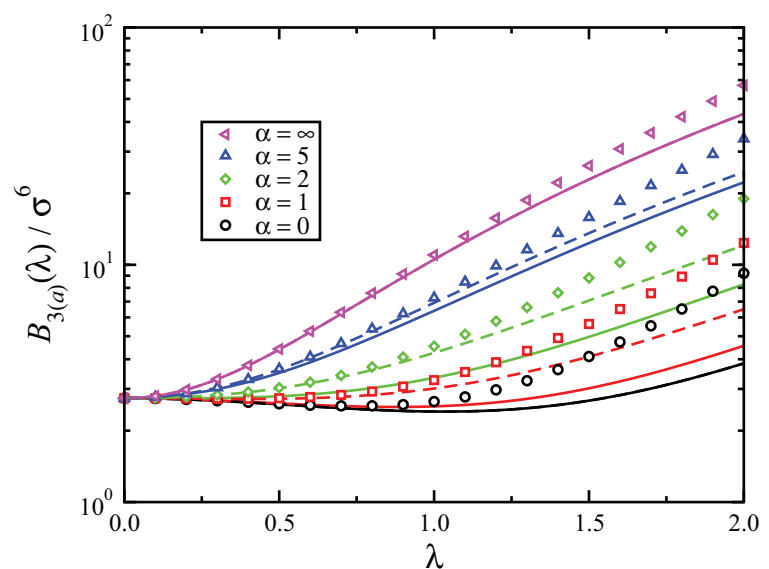

FIG. 4. (Color online) Contributions to the third virial coefficient $B_{3}(\lambda)$ as functions of the dipolar coupling constant $\lambda$ from theory (lines) and Mayer sampling (points) at $\alpha=0$ (black circles and lines), $\alpha=1$ (red squares and lines), $\alpha=2$ (green diamonds and lines), $\alpha=5$ (blue triangles and lines), and $\alpha=\infty$ (magenta left-triangles and lines). The points are $B_{3 a}$ from Mayer sampling, the dashed lines are $B_{3 a}$ from theory, and the solid lines are $B_{3}$ from theory. For $\alpha=0$ and $\alpha=\infty, B_{3}=B_{3 a}$.

no deviation from the results from computations, as shown in Fig. 3. Deviations increase with $\lambda>1$, due to the truncation of the $\lambda$ expansion. Figure 4 shows both $B_{3}$ and $B_{3 a}$ from theory, and $B_{3 a}$ from Mayer sampling; note the logarithmic ordinate and that $B_{3 b}$ is omitted from the plot because it is negative. For $\alpha=0$ and $\alpha=\infty, B_{3}-B_{3 a}=B_{3 b}=0 . B_{3 b}$ is significant for $\alpha=1$ and $\alpha=2$, and rather small for $\alpha=5$, all in correspondence with the results shown in Fig. 2. In relative terms, the theoretical expression for $B_{3 a}$ is more accurate at high values of $\alpha$ than at low values; again, this is evident from Fig. 2 .

In summary, the analytical expressions for $B_{2}$ and $B_{3}$ given by Eqs. (31) and (32), respectively, appear to be extremely reliable for $\lambda=1$, and at least qualitatively correct for $\lambda=2$. The limitation is the truncation of the expansion in $\lambda$ at cubic order, but higher order terms are very difficult to calculate. The dependences of the virial coefficients on $\alpha$ are captured extremely well in all cases.

\section{B. Equation of state}

The virial coefficients are now used to calculate equations of state, presented here as the compressibility factor $Z$ as a function of reduced concentration $\rho^{*}=\rho \sigma^{3}$. Figure 5 shows the equations of state for DHS fluids with $\lambda=1$ and $\alpha=1,2$, 5 , and $\infty$. (Note that the case $\alpha=0$ was considered already in Ref. [24].) Two theoretical expressions are tested against simulation results: the PVE expression [Eq. (36)] of Nezbeda and co-workers; and the LFE expression [Eq. (42)] obtained by the current authors. The inputs to each theory are the same, these being $B_{2}$ and $B_{3}$. With $\alpha=1$ and $\alpha=2$, the PVE and LFE expressions give essentially the same results and are in excellent agreement with the results from MC simulations, over the range $0 \leqslant \rho^{*} \leqslant 0.8$. With $\alpha=5$ and $\alpha=\infty$, the two theories differ substantially, with the PVE expression significantly overestimating the pressure at concentrations 

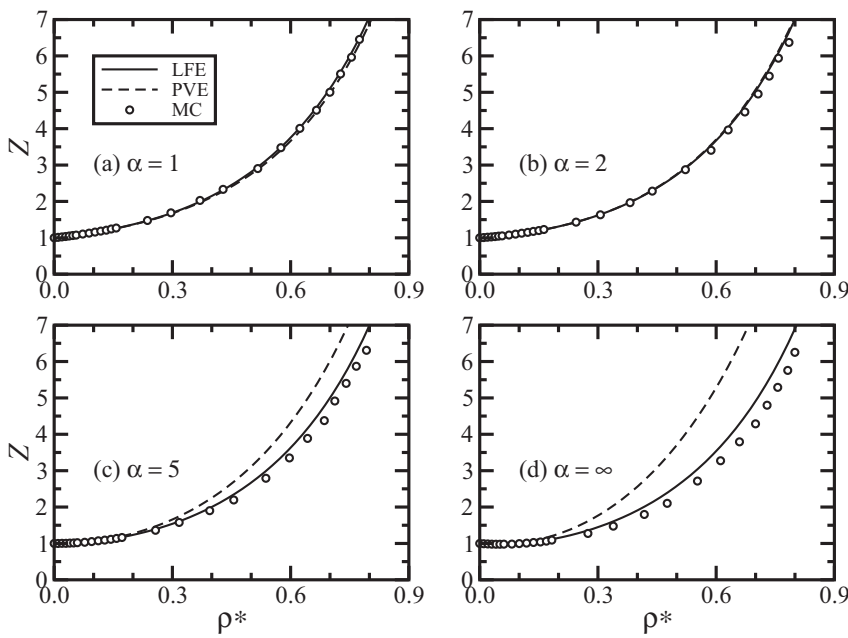

FIG. 5. Compressibility factor $Z=P V / N k_{\mathrm{B}} T$ versus concentration $\rho^{*}=\rho \sigma^{3}$ for a system with dipolar coupling constant $\lambda=1$, from the PVE [Eq. (36)] (dashed lines), the LFE theory [Eq. (42)] (solid lines), and MC simulation (points). Data are shown for Langevin parameters (a) $\alpha=1$, (b) $\alpha=2$, (c) $\alpha=5$, and (d) $\alpha=\infty$.

$\rho^{*} \geqslant 0.3$. The LFE expression, however, continues to track the simulation results all the way up to $\rho^{*}=0.8$, with the maximum deviation in $Z$ being of order $10 \%$.

The equations of state for DHS fluids with $\lambda=2$ and $\alpha=1$, 2,5 , and $\infty$ are shown in Fig. 6 . With $\alpha=1$, the PVE and LFE expressions give almost identical results, and which deviate slightly from the results from MC simulations. The deviations are more pronounced with $\alpha=2,5$, and $\infty$, but while the PVE fails quite badly, the LFE expression continues to track the $\mathrm{MC}$ results.

Overall, the LFE expression gives superior results for $\lambda \leqslant$ 2. This can be traced back to the logarithmic expression for the free energy [Eq. (38)], which contains terms at all orders of $\rho$ when the logarithm is expanded, albeit with coefficients
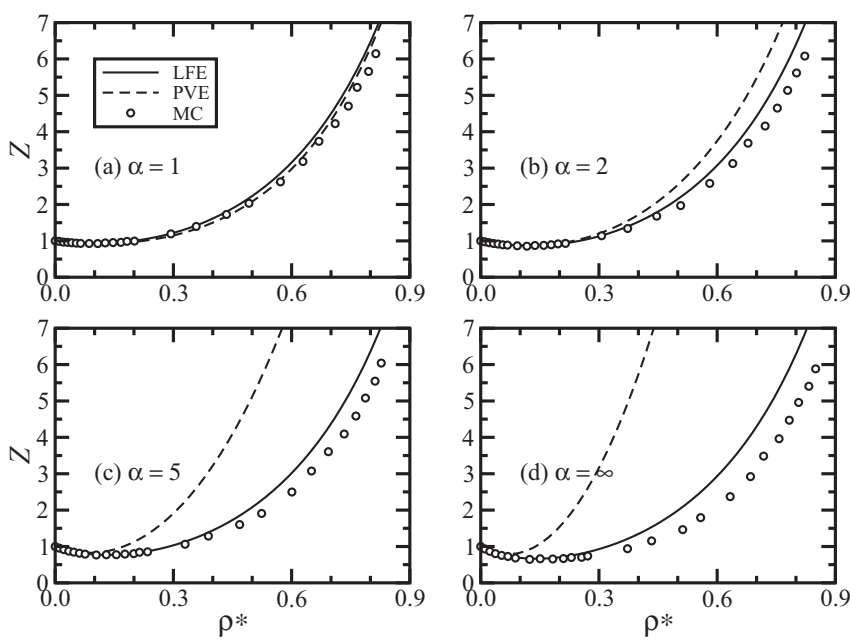

FIG. 6. Compressibility factor $Z=P V / N k_{\mathrm{B}} T$ versus concentration $\rho^{*}=\rho \sigma^{3}$ for a system with dipolar coupling constant $\lambda=2$, from the PVE [Eq. (36)] (dashed lines), the LFE theory [Eq. (42)] (solid lines), and MC simulation (points). Data are shown for Langevin parameters (a) $\alpha=1$, (b) $\alpha=2$, (c) $\alpha=5$, and (d) $\alpha=\infty$.
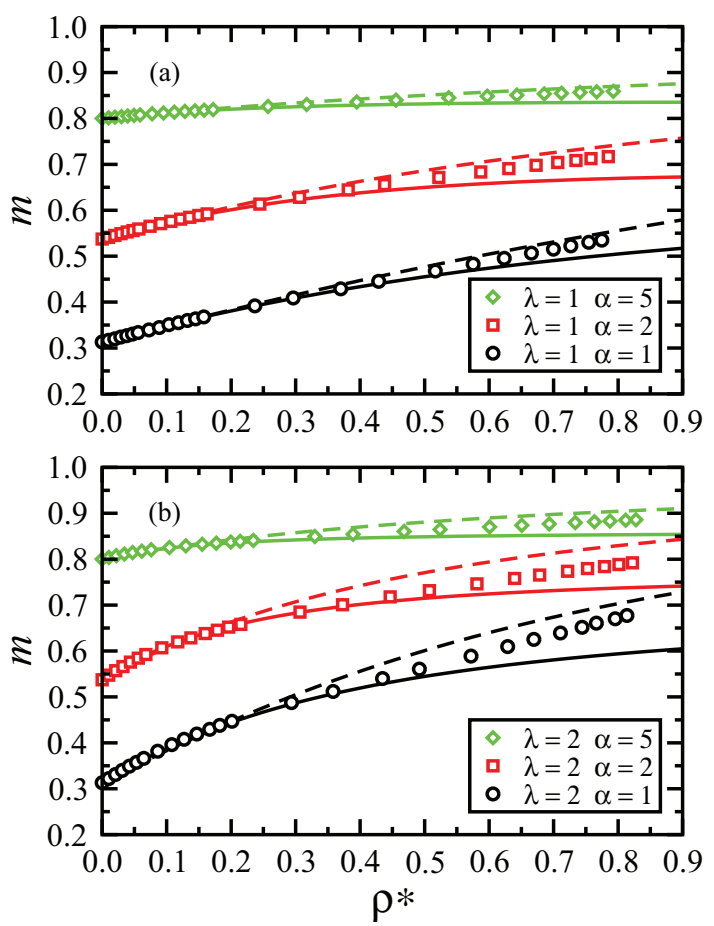

FIG. 7. (Color online) The fractional magnetization $m$ as a function of concentration $\rho^{*}=\rho \sigma^{3}$ for DHS fluids with $\alpha=1,2$, and 5 and (a) $\lambda=1$ and (b) $\lambda=2$. The points are from MC simulations, the solid lines are from LFE theory, and the dashed lines are from MMF2 theory [38].

related to the input values for $B_{2}$ and $B_{3}$. By construction, the low-concentration behavior is captured exactly.

\section{Magnetization}

The fractional (scalar) magnetization $m$ is given by

$$
m=\frac{1}{N \mu}\left|\left\langle\sum_{i=1}^{N} \boldsymbol{\mu}_{i}\right\rangle\right|=-\frac{\partial}{\partial \alpha}\left(\frac{\beta F}{N}\right) .
$$

At very low concentrations, $\beta F / N=\ln (\rho \mathcal{V} / \Psi)-1$ [Eq. (38)] and hence $m=\operatorname{coth}(\alpha)-\alpha^{-1}$, the Langevin singleparticle result. Changes in $m$ with increasing concentration are due to increasing interparticle correlations. Figure 7 shows $m$ as a function of $\rho^{*}$ for DHS fluids with $\lambda=1$ and 2, and $\alpha=1,2$, and 5, from MC simulations and theory. The magnetization is obtained from the LFE theory by inserting the $\alpha$-dependent virial coefficients in to Eqs. (38) and (48). Also shown in Fig. 7 are the predictions of the MMF2 theory of Ivanov and Kuznetsova [38]. All of the results show that interparticle correlations give rise to substantial changes in magnetization as the concentration is increased from zero up to 0.85 , particularly with low values of $\alpha$. Both of the LFE and MMF2 theories are in essentially perfect agreement with the MC simulation results up to $\rho^{*} \simeq 0.2$. The LFE theory continues to work well up to $\rho^{*} \simeq 0.45$ (as it did in the equations of state). At higher concentrations, neither theory is perfect, but the MMF2 tracks the MC simulation results more closely. The LFE theory may be convenient for analyzing the magnetization curves of real ferrofluids at volume fractions up to $\varphi \simeq 0.2\left(\rho^{*} \simeq 0.4\right)$, because the effects 

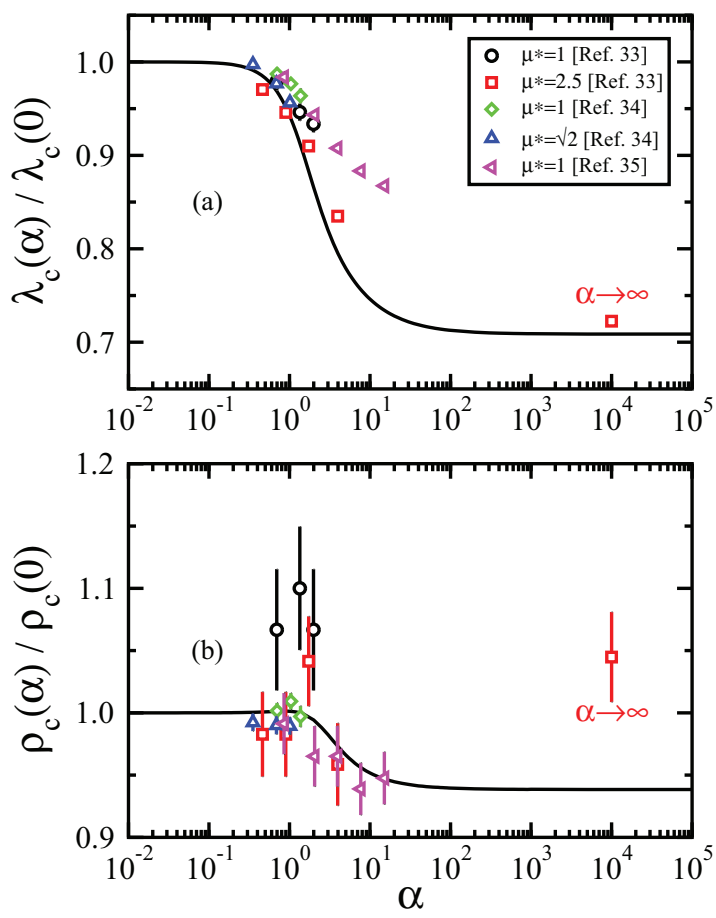

FIG. 8. (Color online) Relative variations of (a) the critical dipolar coupling constant $\lambda_{\mathrm{c}}$ and (b) the critical concentration $\rho_{\mathrm{c}}$ with the Langevin parameter $\alpha$, compared to the zero-field values. The points are from simulations of Stockmayer fluids with reduced dipole moments of $\mu^{*}=1$ [44-46], $\mu^{*}=\sqrt{2}$ [45], and $\mu^{*}=2.5$ [44], while the curves are from the LFE theory. The simulation points for $\mu^{*}=2.5$ and $\alpha=\infty$ from Ref. [44] are shown at $\alpha=10^{4}$.

of additional, nonmagnetic interactions can be incorporated straightforwardly into the virial coefficients.

\section{Phase behavior}

As explained in the Introduction, DHSs apparently do not undergo a condensation transition, either in zero field or in an applied field. Nonetheless, almost all liquid-state theories (virial expansion, thermodynamic perturbation theory, integral equations, DFT) predict such a transition. The presence of additional, isotropic interactions does lead to a transition, even in zero field, and the critical parameters $\left(\rho_{\mathrm{c}}\right.$ and $\left.T_{\mathrm{c}}\right)$ can be traced as functions of the applied field. For example, the critical parameters of the Stockmayer fluid have been measured as functions of the field strength in computer simulations [44-46]. Figure 8 shows critical parameters in the form $\lambda_{\mathrm{c}}(\alpha) / \lambda_{\mathrm{c}}(0)$ and $\rho_{\mathrm{c}}(\alpha) / \rho_{\mathrm{c}}(0)$ from simulations of the Stockmayer fluid with different values of the reduced dipole moment, $\mu^{*}=\sqrt{\mu_{0} \mu^{2} / 4 \pi \sigma_{\mathrm{LJ}}^{3} \epsilon_{\mathrm{LJ}}}$, where $\sigma_{\mathrm{LJ}}$ and $\epsilon_{\mathrm{LJ}}$ are the Lennard-Jones potential parameters. The relative changes in critical parameters from the LFE theory are also shown, these being obtained from the solutions of the simultaneous equations $(\partial P / \partial V)_{T}=\left(\partial^{2} P / \partial V^{2}\right)_{T}=0$. (For $\alpha=0, \lambda_{\mathrm{c}}=4.54$ and $\rho_{\mathrm{c}}^{*}=0.0978$; for $\alpha=\infty, \lambda_{\mathrm{c}}=3.21$ and $\rho_{\mathrm{c}}^{*}=0.0917$.) The results from simulations and theory are in excellent agreement, particularly for $\mu^{*}=2.5$ where the dipolar interaction dominates over the Lennard-Jones attraction; this shows that the LFE is describing accurately the dipolar interactions in applied fields. The critical dipolar coupling constant decreases with increasing $\alpha$, meaning that the critical temperature increases. This is due to the decrease in $B_{2}$ (signaling more attractive interactions) dominating over the increase in $B_{3}$ (signaling more repulsive interactions). The increase in critical temperature between $\alpha=0$ and $\alpha=\infty$ is about $40 \%$. The critical concentration varies only weakly with $\alpha$ : the simulation results are not in agreement with each other, while the theory predicts a slight decrease of about $6 \%$ between $\alpha=0$ and $\alpha=\infty$. It is stressed that the theory contains dipolar interactions only, and that for a proper quantitative comparison, the isotropic (Lennard-Jones) interactions should be included. DFT calculations on the Stockmayer fluid by Groh and Dietrich show equally good agreement with simulations, at least in terms of the relative critical temperature [30]. Note, however, that DFT calculations require numerical work to obtain the equilibrium orientational distribution function, whereas in the current theory, thermodynamic calculations are trivial once the virial coefficients have been determined. Moreover, the DFT calculations predict the existence of a critical point associated with a transition between isotropic and ferromagnetic liquid phases; the homogeneous ferromagnetic liquid phase in zero field has not been observed experimentally, although it has been observed in simulated finite-size systems [63-68]. Nonetheless, Groh and Dietrich's DFT calculations represent probably the best available description of the vaporliquid transition in Stockmayer fluids in an applied field.

In recent experiments, Socoliuc et al. found indications of a putative field-dependent condensation transition in an aqueous ferrofluid, containing magnetite nanoparticles with diameter $\sigma \simeq 5 \mathrm{~nm}$ [69]. Experimental observations suggest that there is a field-dependent critical temperature $T_{\mathrm{c}}(H)$ below which large concentrated droplets of magnetic particles coexist with a dilute phase. These droplets were observed to be shaped like prolate ellipsoids aligned with the magnetic field and to have dimensions on the order of $\mu \mathrm{m}$. The critical temperature was seen to increase with increasing field strength and reach an asymptotic value of around $313 \mathrm{~K}$. There are some questions as to the effects of polydispersity and clustering of particles driven by nonmagnetic (van der Waals and hydrophobic) interactions, but the experimental measurements are at least in qualitative agreement with the results presented in Fig. 8.

\section{CONCLUSIONS}

In this work the thermodynamic properties of dipolar hard sphere fluids in applied fields have been examined using a combination of theory and computer simulation. This model is known to provide a reliable representation of ferrofluids. Analytical results for the second and third virial coefficients have been obtained in terms of an expansion in the dipolar coupling constant $\lambda$ and appropriate functions of the Langevin parameter $\alpha$. The exact formula for the third virial coefficient has been derived, and it differs from the usual case of zero applied field. The analytical formulas have been compared against numerical results from Mayer-sampling calculations. For the realistic values $\lambda \leqslant 2$, the analytical formulas capture the $\alpha$ dependences of the virial coefficients essentially exactly. For $\lambda=1$ the agreement between the formulas and numerical results is excellent, while for $\lambda=2$, deviations set in due to the truncation of the expansion in $\lambda$. With increasing 
applied field, the second virial coefficient, $B_{2}$, decreases (signaling an increase in attractive interactions) while the third virial coefficient, $B_{3}$, increases (signaling increasing repulsive interactions). These trends have been rationalized in terms of the increasing contribution to $B_{2}$ from attractive nose-to-tail parallel interactions between pairs of dipoles, and the increasing contribution to $B_{3}$ from repulsive side-by-side parallel interactions between dipoles in the majority of possible three-particle configurations.

The virial coefficients have been incorporated in to various forms of virial expansion for the equation of state and compared to results from Monte Carlo simulations. Two thermodynamic theories have been tested: the "perturbed virial expansion" of Nezbeda and co-workers [35-37], which in the present case is based on the difference between the virial expansions of hard spheres and dipolar hard spheres (truncated at the third virial coefficient); and the "logarithmic free energy" theory of the current authors, in which the virial expansion of the Helmholtz free energy is resummed in to a logarithmic form. For $\lambda \leqslant 2$ it was shown that the logarithmic free energy theory is superior. This is due to the approximate incorporation of higher order terms in the concentration expansion. In future work, it would be interesting to study the high-density properties of the DHS fluid, and to accommodate any well-defined, limiting behavior in the logarithmic free energy expression. Calculations of this type have been performed successfully for soft-sphere and hard-sphere fluids [70]. One potential complication is the formation of long-range orientationally ordered phases in DHS fluids at high density and low temperature [63-68].
The logarithmic free energy theory also yields the magnetization by differentiation with respect to $\alpha$. A comparison of the theory with computer simulation results, and the second-order modified mean-field theory of Ivanov and Kuznetsova [38], shows excellent agreement at low-to-moderate concentrations. At high concentrations, both theories deviate from the simulation results. Nonetheless, the new theory has the merit that nonmagnetic interactions can easily be incorporated in to the virial coefficients.

Finally, predictions of the critical parameters for the condensation transition have been obtained from the logarithmic free energy theory and compared with computer-simulation results. Although dipolar hard spheres do not undergo such a transition, systems with an additional, isotropic attraction do. Computer simulations of the Stockmayer fluid have yielded field-dependent critical parameters [44-46]. A comparison of the relative changes in critical parameters with increasing field strength shows very good agreement, indicating that the theory is handling the dipolar interactions correctly. Further work to incorporate the nondipolar interactions is in progress.

\section{ACKNOWLEDGMENTS}

E.A.E. and A.O.I. gratefully acknowledge grants from the Ministry of Education and Science of the Russian Federation (N2.609.2011) and the Russian Foundation for Basic Research (N12-02-31079, N13-02-91052-CNRS-a). E.A.E. and P.J.C. thank the Ural Federal University for supporting collaborative visits between Edinburgh and Ekaterinburg.
[1] R. E. Rosensweig, Ferrohydrodynamics (Dover Publications, New York, 1998).

[2] E. A. Elfimova, A. O. Ivanov, and P. J. Camp, J. Chem. Phys. 136, 194502 (2012)

[3] A. O. Ivanov, S. S. Kantorovich, E. N. Reznikov, C. Holm, A. F. Pshenichnikov, A. V. Lebedev, A. Chremos, and P. J. Camp, Phys. Rev. E 75, 061405 (2007).

[4] A. O. Ivanov, S. S. Kantorovich, E. N. Reznikov, C. Holm, A. F. Pshenichnikov, A. V. Lebedev, A. Chremos, and P. J. Camp, Magnetohydrodynamics 43, 393 (2007).

[5] P. G. de Gennes and P. A. Pincus, Phys. Kondens. Materie 11, 189 (1970).

[6] L. Rovigatti, J. Russo, and F. Sciortino, Phys. Rev. Lett. 107, 237801 (2011).

[7] L. Rovigatti, J. Russo, and F. Sciortino, Soft Matter 8, 6310 (2012).

[8] J.-M. Caillol, J. Chem. Phys. 98, 9835 (1993).

[9] J. J. Weis and D. Levesque, Phys. Rev. Lett. 71, 2729 (1993).

[10] P. J. Camp, J. C. Shelley, and G. N. Patey, Phys. Rev. Lett. 84, 115 (2000).

[11] S. C. McGrother, D. C. Williamson, and G. Jackson, J. Chem. Phys. 104, 6755 (1996).

[12] I. Szalai, D. Henderson, D. Boda, and K.-Y. Chan, J. Chem. Phys. 110, 7348 (1999).

[13] G. Ganzenmüller and P. J. Camp, J. Chem. Phys. 126, 191104 (2007).
[14] N. G. Almarza, E. Lomba, C. Martín, and A. Gallardo, J. Chem. Phys. 129, 234504 (2008).

[15] Y. V. Kalyuzhnyi, I. A. Protsykevytch, G. Ganzenmüller, and P. J. Camp, Europhys. Lett. 84, 26001 (2008).

[16] M. Martín-Betancourt, J. M. Romero-Enrique, and L. F. Rull, J. Phys. Chem. B 113, 9046 (2009).

[17] G. Ganzenmüller, G. N. Patey, and P. J. Camp, Mol. Phys. 107, 403 (2009).

[18] R. P. Sear, Phys. Rev. Lett. 76, 2310 (1996).

[19] R. van Roij, Phys. Rev. Lett. 76, 3348 (1996).

[20] J. M. Tavares, M. M. Telo da Gama, and M. A. Osipov, Phys. Rev. E 56, R6252 (1997).

[21] Y. Levin, Phys. Rev. Lett. 83, 1159 (1999).

[22] G. Stell, J. C. Rasaiah, and H. Narang, Mol. Phys. 23, 393 (1972).

[23] G. Stell, J. C. Rasaiah, and H. Narang, Mol. Phys. 27, 1393 (1974).

[24] E. A. Elfimova, A. O. Ivanov, and P. J. Camp, Phys. Rev. E 86, 021126 (2012).

[25] B. Luigjes, D. M. E. Thies-Weesie, A. P. Philipse, and B. H. Erné, J. Phys.: Condens. Matter 24, 245103 (2012).

[26] B. Luigjes, D. M. E. Thies-Weesie, B. H. Erné, and A. P. Philipse, J. Phys.: Condens. Matter 24, 245104 (2012).

[27] J. B. Hayter and R. Pynn, Phys. Rev. Lett. 49, 1103 (1982).

[28] S. Klapp and F. Forstmann, Phys. Rev. E 60, 3183 (1999).

[29] S. H. L. Klapp and G. N. Patey, J. Chem. Phys. 112, 3832 (2000). 
[30] B. Groh and S. Dietrich, Phys. Rev. E 53, 2509 (1996).

[31] E. A. Elfimova and A. O. Ivanov, J. Exp. Theor. Phys. 111, 146 (2010).

[32] S. Caracciolo, B. M. Mognetti, and A. Pelissetto, J. Chem. Phys. 125, 094903 (2006).

[33] S. Caracciolo, B. M. Mognetti, and A. Pelissetto, Macromol. Theory Simul. 17, 67 (2008).

[34] J. K. Singh and D. A. Kofke, Phys. Rev. Lett. 92, 220601 (2004).

[35] K. Aim and I. Nezbeda, Fluid Phase Equil. 12, 235 (1983).

[36] I. Nezbeda and W. R. Smith, Fluid Phase Equil. 216, 183 (2004).

[37] J. Krejci and I. Nezbeda, Fluid Phase Equil. 314, 156 (2012).

[38] A. O. Ivanov and O. B. Kuznetsova, Phys. Rev. E 64, 041405 (2001).

[39] A.-P. Hynninen and M. Dijkstra, Phys. Rev. Lett. 94, 138303 (2005).

[40] B. Smit and D. Frenkel, Mol. Phys. 68, 951 (1989).

[41] M. E. van Leeuwen and B. Smit, Phys. Rev. Lett. 71, 3991 (1993).

[42] M. E. van Leeuwen, B. Smit, and E. M. Hendriks, Mol. Phys. 78, 271 (1993).

[43] M. E. van Leeuwen, Mol. Phys. 82, 383 (1994).

[44] M. J. Stevens and G. S. Grest, Phys. Rev. E 51, 5976 (1995).

[45] D. Boda, B. Kalmár, J. Liszi, and I. Szalai, J. Chem. Soc., Faraday Trans. 92, 2709 (1996).

[46] T. Kristóf, J. Liszi, and I. Szalai, Phys. Rev. E 69, 062106 (2004).

[47] J. Bartke and R. Hentschke, Phys. Rev. E 75, 061503 (2007).

[48] G. Ganzenmüller and P. J. Camp, J. Chem. Phys. 127, 154504 (2007).

[49] R. Hentschke, J. Bartke, and F. Pesth, Phys. Rev. E 75, 011506 (2007).

[50] A. O. Ivanov, S. S. Kantorovich, and P. J. Camp, Phys. Rev. E 77, 013501 (2008).
[51] R. Hentschke and J. Bartke, Phys. Rev. E 77, 013502 (2008).

[52] R. Jia and R. Hentschke, Phys. Rev. E 80, 051502 (2009).

[53] J.-P. Hansen and I. R. McDonald, Theory of Simple Liquids, 3rd ed. (Academic Press, London, 2006).

[54] M. J. Stevens and G. S. Grest, Phys. Rev. Lett. 72, 3686 (1994).

[55] M. J. Stevens and G. S. Grest, Phys. Rev. E 51, 5962 (1995).

[56] R. Jia, H. Braun, and R. Hentschke, Phys. Rev. E 82, 062501 (2010).

[57] S. Dussi, L. Rovigatti, and F. Sciortino, Mol. Phys., doi: 10.1080/00268976.2013.838315.

[58] K. R. S. Shaul, A. J. Schultz, and D. A. Kofke, J. Chem. Phys. 135, 124101 (2011).

[59] T. L. Hill, Statistical Mechanics: Principles and Selected Applications (Dover Publications, New York, 1987).

[60] See Supplemental Material at http://link.aps.org/supplemental/ 10.1103/PhysRevE.88.042310 for details of the $\lambda$ expansion of $B_{2}$ and $B_{3}$.

[61] N. F. Carnahan and K. E. Starling, J. Chem. Phys. 51, 635 (1969).

[62] M. P. Allen and D. J. Tildesley, Computer Simulation of Liquids (Clarendon Press, Oxford, 1987).

[63] D. Wei and G. N. Patey, Phys. Rev. Lett. 68, 2043 (1992).

[64] D. Wei and G. N. Patey, Phys. Rev. A 46, 7783 (1992)

[65] J. J. Weis and D. Levesque, Phys. Rev. E 48, 3728 (1993).

[66] J.-J. Weis, J. Chem. Phys. 123, 044503 (2005).

[67] J.-J. Weis and D. Levesque, J. Chem. Phys. 125, 034504 (2006).

[68] M. A. Pounds and P. A. Madden, J. Chem. Phys. 126, 104506 (2007).

[69] V. Socoliuc, D. Bica, and L. Vékás, Magnetohydrodynamics 47, 201 (2011).

[70] N. S. Barlow, A. J. Schultz, S. J. Weinstein, and D. A. Kofke, J. Chem. Phys. 137, 204102 (2012). 